\title{
QoS Analysis for Web Service Compositions Based on Probabilistic QoS
}

\author{
Huiyuan Zheng ${ }^{1}$, Jian Yang ${ }^{1}$, Weiliang Zhao ${ }^{1}$, and Athman Bouguettaya ${ }^{2}$ \\ 1 Department of Computing, Macquarie University, Australia \\ \{huiyuan.zheng, jian.yang, weiliang.zhao\}@mq.edu. au \\ 2 School of Computer Science and Information Technology, RMIT University, \\ Australia \\ athman.bouguettaya@rmit.edu.au
}

\begin{abstract}
Quality of Service (QoS) analysis and prediction for Web service compositions is an important and challenging issue in distributed computing. In existing work, QoS for service compositions is either calculated based on constant QoS values or simulated based on probabilistic QoS distributions of component services. Simulation method is time consuming and can not be used in real-time applications for dynamic Web service compositions. In this paper, we propose a calculation method to estimate the QoS of a service composition, in which the probability distributions of the QoS of component services can be in any shape. Experimental results show that the proposed QoS calculation approach significantly improves the efficiency in probabilistic QoS estimation.
\end{abstract}

\section{Introduction}

Web services technology creates the opportunity for building composite services by combining existing elementary or complex services (i.e. the component services) from different enterprises and in turn offering them as high-level services or processes (i.e. the composite services) 13. QoS analysis becomes increasingly challenging and important when complex and mission critical applications are built upon services with different QoS. Thus solid model and method support for QoS predication in service composition becomes crucial in further analysis of complexity and reliability in developing service oriented distributed applications.

The QoS of a Web service is specified in service level agreement (SLA) between the service provider and service consumers. Most QoS in SLA are expressed as constant values 14. Recently, probabilistic QoS has gained attentions because of the dynamic and unpredictable nature of Internet and its benefits have been recognized as being accurate and flexible 8511. With QoS modeled as probability distributions, service clients can have a better understanding of the performance of a service, and they can thereby have a relatively precise perception of the QoS of a composite service. A service provider also benefits from probabilistic QoS in SLA, because setting QoS as constant values in SLA does not reflect the dynamic characteristics of certain QoS metrics and may lead to pessimistic contracts [11].

G. Kappel, Z. Maamar, H.R. Motahari-Nezhad (Eds.): ICSOC 2011, LNCS 7084, pp. 47-61 2011.

(C) Springer-Verlag Berlin Heidelberg 2011 
When the QoS of its component services are modeled as probability distributions, exiting work adopts simulation approach to compute the QoS distribution for a composite service [11. Simulation method is time consuming. It works fine if used in design time when the architecture and component Web services of a service composition are determined. However, the service environment can be dynamic. Service-based processes should dynamically change to adapt to this environment. Composition engines, such as e-flow [4] and SELF-SERV [2], are designed for the purpose of run time composition. In these composition engines, QoS of the composite service needs to be estimated in real-time. Simulation method for QoS estimation will become a bottle-neck in real-time scenarios.

In order to overcome the problems mentioned above, we propose a method to estimate QoS for composite services. The basic idea is: (1) a QoS metric (e.g. execution time) of a component service is modeled as a probability distribution; (2) A composite service is modeled as a service graph and is composed of four basic patterns as Sequential, Parallel, Conditional, and Loop. QoS aggregation operations are defined and formulae are developed to compute QoS probability distributions for these patterns; (3) QoS calculation for a composite service becomes a matter of iteratively applying the QoS aggregation operations for these composite patterns. In comparison with the existing work, the contributions of this paper can be summarized as follows:

- In the proposed method, QoS for a composite service is calculated, not simulated. Experiments show that the proposed method is not only accurate but also much more efficient than simulation approach.

- The proposed method provides a more general and systematic approach compared with existing methods. As a result, the problems dealt with in the existing methods for QoS aggregation become the special cases in the proposed method. In this work, we do not have any assumptions on the forms of the QoS distributions, i.e., they can be any shaped probability distributions.

In the rest of the paper we will use the term component $Q o S$ and composite QoS to refer to QoS of component service and QoS of composite service respectively. We will also use QoS and QoS metric interchangeably.

The remainder of the paper is organized as follows: Section 2 discusses the work related to QoS estimation. In Section 3, the method of modeling and processing the structure for a composite service is introduced. In Section 4, QoS calculation method for Web service compositions is provided. Experiments are carried out in Section 5. Section [6] concludes the paper.

\section{Related Work}

We will first review QoS modeling method for Web services. Then, QoS monitoring methods for Web services will be summarized. Through these QoS monitoring methods, history QoS, i.e. QoS sample data, of a Web service can be obtained. The QoS sample data is a source of generating probability distributions for Web 
services. Finally, current QoS estimation methods for service compositions will be discussed.

QoS Models: Existing research in service QoS representation can be categorized as: single values representation, multiple values representation, and standard statistical distributions. In most work, each QoS metric is represented as a constant value [714. As the QoS of a Web service changes with time and environment settings, single value-modeled QoS does not reflect this variation. Standard statistical distributions are adopted to model QoS to solve the problem 311. 33 mentions that a QoS metric can be specified as a distribution function, such as Exponential, Normal, Weibull, and Uniform. 11] argues that the contracts between Web service provider and client can be expressed as QoS probability distributions. T location-scale distribution is adopted to fit the original monitored QoS data of Web services. However, the reality is that an actual QoS probability distribution can come in any shape, which may not be able to fit into any well known statistical distributions. A more precise and general QoS modeling method has been proposed in our previous work [16], which is basically a free shaped probability distribution.

QoS Monitoring: The QoS of a Web service can be obtained through QoS monitoring. There are three strategies for QoS monitoring depending on where the measurement takes place: (1) Client-side monitoring: the measurement of QoS is run on the client side 912 . QoS metric that depends on user experience, such as response time, can be measured on the client side. (2) Server-side monitoring: the measurement of QoS is run on the server side [1]. This technique requires access to the actual Web service implementation, which is not always possible in practice. (3)Third party based monitoring: the measurement of QoS is run on a third party [18. Third parties will periodically probes the service from different geographic locations under various network conditions and generate the QoS.

Composite QoS Aggregation: For single values represented QoS, aggregation method [37] is proposed to calculate the composite QoS. A composition can be regarded as being composed of different composition patterns. Formulae to calculate QoS for these patterns are given. But these formulae can only be applied to single values. For multiple values represented QoS [6], the calculation method is pretty much the same as it is for single values, except that the probability of each QoS value of the composite service are taken into account. For standard distribution represented QoS 311, simulation approaches are applied to estimate the composite QoS. A simulation needs to be run for thousands of times before a QoS sample for the composite service can be obtained. Simulation method is time consuming. An efficient method is necessary for estimating the QoS probability distributions of composite services, which is the focus of this paper.

Our previous work [151617] provide solutions to problems in QoS analysis of Web service compositions. [17] proposes a modeling method for Web service compositions and composition patterns, as well as a QoS analysis method for Web service compositions whose component QoS are modeled as constant values. 15 designs an algorithm to explore the process of a Web service composition with 
complex structures, such as unstructured loop patterns and nested composition patterns. Examples and experiments in [16] show that the inaccurate modeling of the QoS for component Web services can generate misleading QoS analysis results for Web service compositions. Discussion has been given on how to model the QoS probability distribution of a Web service accurately so that the QoS of a Web service composition can be estimated precisely. This paper gives a detailed solution to analyzing the QoS for Web service compositions whose component QoS are modeled as probability distributions. Experiments have been done to show the efficiency of our method over the existing ones. The techniques in 15 16 17] and this paper compose a systematical approach to analyzing the QoS for Web service compositions, which can be seen further in Section 3.

\section{Preliminaries}

A composite service can be built up based on four basic composition patterns: Sequential Pattern, Parallel Pattern, Conditional Pattern, and Loop Pattern. By recursively replacing patterns with single nodes having the same QoS as the composition patterns, a composite service will finally be represented by one node and the QoS of this node is the QoS of the composite service. Based on the QoS estimation method described above, it can be seen that three techniques are needed to compute the QoS for a Web service composition: (1) A modeling method for composite services and composition patterns; (2) A QoS calculation method for the four basic composition patterns; (3) An algorithm to explore the model of a composite service, identify composition patterns, calculate the QoS for the patterns, replace the patterns with the nodes with equivalent QoS, and finally get the QoS for the composite service.

The solutions for (1) and (3) have been given in our previous work [17] and 15] respectively. We will briefly summarize the solutions for (1) and (3) in this section, then we will focus on (2), i.e., the QoS calculation method for composition patterns, in the next section.

\subsection{Modeling Composite Services and Composition Patterns}

A composite service is modeled as a Service Graph (see Figure2 for an example of a Service Graph), in which each vertex represents a component service and each arc denotes a transition from one component service to another under a certain probability. Composition patterns (see Table 1 and Figure 1 for descriptions of the patterns) can be defined based on the definition of the Service Graph. The formal notations of a Service Graph and composition patterns are given in [17.

\subsection{Model Processing Algorithm for Composite QoS Computation}

In this paper, we will adopt the algorithm designed in our previous work 15 to calculate the QoS for a composite service. The input of the algorithm is the 


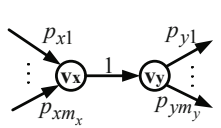

(a) Sequential Pattern

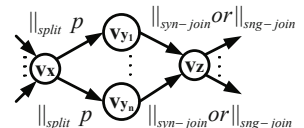

(b) Parallel Pattern

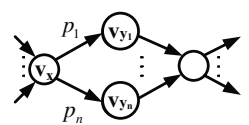

(c) Conditional Pattern

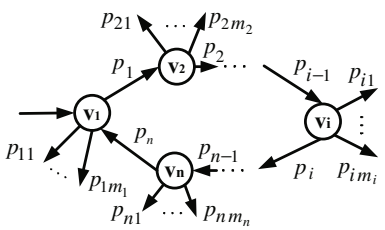

(d) Loop Pattern

Fig. 1. Basic Composition Patterns

Table 1. Descriptions for Composition Patterns

\begin{tabular}{|c|c|c|}
\hline Pattern & & Description \\
\hline Sequential & $\begin{array}{l}\text { In a Sequenti } \\
\text { immediately a }\end{array}$ & $\begin{array}{l}\text { al Pattern (see Figure } 1(\mathrm{a}) \mathrm{e} \text {, a Web service is invoked } \\
\text { fter the completion of a preceding Web service. }\end{array}$ \\
\hline \multirow[t]{2}{*}{ Parallel } & $\begin{array}{l}\text { Synchronized } \\
\text { merge: }\end{array}$ & $\begin{array}{l}\text { In a Parallel Pattern with synchronized merge (see Figure } \\
1(\mathrm{~b}) \text { in which ' } \|_{\text {syn-join }} \text { 'represents synchronized merge), } \\
\text { Web services are executed concurrently. The subsequent } \\
\text { Web service will be invoked when all the Web services in } \\
\text { the parallel pattern have finished running. }\end{array}$ \\
\hline & Single merge: & $\begin{array}{l}\text { In a Parallel Pattern with single merge (see Figure } 1(\mathrm{~b}) \text { in } \\
\text { which ' } \|_{\text {sng-join' }} \text { represents single merge), Web services } \\
\text { are executed concurrently. The subsequent Web service } \\
\text { will be invoked when one of the Web services in the par- } \\
\text { allel pattern has finished running. }\end{array}$ \\
\hline Conditional & \multicolumn{2}{|c|}{$\begin{array}{l}\text { In a Conditional Pattern (see Figure } 1(\mathrm{c}) \mathrm{d} \text {, the Web services are run } \\
\text { exclusively. }\end{array}$} \\
\hline Loop & \multicolumn{2}{|c|}{$\begin{array}{l}\text { In a Loop Pattern (see Figure } 1(\mathrm{~d})] \text {, the Web services are run repeat- } \\
\text { edly. A Loop Pattern has more than one entry or exit point. }\end{array}$} \\
\hline
\end{tabular}

Service Graph of a composite service, as well as the QoS of the component services. The output of the algorithm is the QoS for the composite service.

Different from [15] in which the QoS are single values, the QoS in this paper are probability distributions. Therefore, the QoS calculation methods for composition patterns in the algorithm should be changed accordingly, which will be discussed in detail in the following section.

\section{Probabilistic QoS Aggregation}

QoS aggregation formulae are developed in this section to calculate the QoS for composition patterns. 


\subsection{Approach Overview and Underlying Assumptions}

A QoS metric of each service (either a component service or a composite service) is seen as a random variable and the following assumptions have been made:

(1) The Qo: 11 of different component services are mutually independent, i.e. the QoS of any two component services are independent. If the QoS of two services are independent, the QoS of one service does not affect the QoS of the other service.

(2) QoS control is out of the scope of this paper. We only consider the case that the developer of a composite service makes use of the component services but has no control of the QoS of the component services. The QoS probability distributions of a component service are statistically estimated and have already taken into account different QoS influencing factors such as workload.

(3) The transition probabilities from one service to another in a composite service can either be provided according to the experience of the service developer at design time or be statistically estimated based on the execution history of the service. Detailed method of obtaining the transition probabilities in a composite service can be found in [3].

(4) QoS are represented as histograms with the same start point and width of intervals. There is more accurate method of getting the QoS probability distribution based on a QoS sample, which is out of the scope of this paper and can be found in [16].

\subsection{QoS Probability Distribution Calculation for Composition Patterns}

Classification of QoS Metrics. The QoS metrics are classified into five categories according to their characteristics in different composition patterns, which are: additive, multiplicative, concave (i.e. minimum), convex (i.e. maximum), and weighted additive. For example, the QoS metric execution time reflects an additive behavior in a Sequential Pattern and convex behavior in a Parallel Pattern with synchronized merge. In this paper, the discussion of QoS analysis is based on these categories instead of individual QoS metrics, which makes the QoS analysis approach more general and fits more QoS metrics.

Examples of additive, multiplicative, concave, and convex QoS metrics are cost, reliability, execution time of a Parallel Pattern with single merge, and execution time of a Parallel Pattern with synchronized merge respectively.

It is worth mentioning that multiplicative QoS metrics such as availability, reliability, and accessibility, are represented as a statistical percentage value (e.g. 90\%) rather than a distribution. Therefore, only four types of QoS metrics: additive, concave, convex, and weighted additive will be discussed for the computation of composite QoS distribution in the rest of the paper.

QoS Calculation Operations. If the component QoS is represented by single value, to calculate the composite QoS, operations are sum, minimum, maximum, and weighted sum for additive, concave, convex, and weighted additive

\footnotetext{
${ }^{1}$ Unless indicated otherwise, QoS is referred as the same QoS metric.
} 
QoS metrics respectively. However, the computation of the QoS distribution for a composition pattern based on probability-distribution represented component QoS is far more complex than based on single-value represented component QoS. We thus define four operations on probability distributions, which are: QoSSum, QoSMin, QoSMax, and QoSWeightedSum, to distinguish the arithmetic operations on single values.

- QoSSum(denoted as $\circledast)$ : operates on the component QoS distributions taking into consideration of the addition of their QoS values;

- QoSMin(denoted as $₫)$ : operates on the component QoS distributions taking into consideration of the minimum of their QoS values;

- QoSMax (denoted as $\oslash)$ : operates on the component QoS distributions taking into consideration of the maximum of their QoS values;

- QoSWeightedSum(denoted as $\odot$ ): operates on the component QoS distributions taking into consideration of the addition of their QoS values with path probabilities as weights. It is mainly used in Conditional and Loop Patterns.

These operations and their relationships with composition patterns and QoS metrics are summarized in Table 2 .

Table 2. Operations for QoS Aggregation

\begin{tabular}{|c|l|l|}
\hline Pattern & Operation & QoS Metric \\
\hline \hline \multirow{2}{*}{ Sequential } & QoSSum & Additive \\
\cline { 2 - 3 } & QoSMin & Concave \\
\hline \multirow{3}{*}{ Parallel } & QoSSum & Additive \\
\cline { 2 - 3 } & QoSMin(single-merge) & Concave \\
\cline { 2 - 3 } & QoSMax(synchronized-merge) & Convex \\
\hline Conditional & QoSWeightedSum & any \\
\hline \multirow{2}{*}{ Loop } & QoSSum\&QoSWeightedSum & Additive \\
\cline { 2 - 3 } & QoSMin\&QoSWeightedSum & Concave \\
\hline
\end{tabular}

Formulae are developed for these operations. We introduce the following naming conventions:

- $q$ is a variable representing a QoS metric;

- $f(q)$ denotes the density function of the probability distribution (PDF);

- $F(q)$ denotes the cumulative distribution function (CDF); $F(q)$ and $f(q)$ have the following cumulative relationship: $F(q)=\int_{-\infty}^{q} f(x) d x$ for continuous distributions or $F(q)=\sum_{q_{i}<=q} f\left(q_{i}\right)$ for discrete distributions.

It should be noted that although the discussion is based on distributions, the developed formulae are also applied to single values. This is because single values can also be represented as distributions with the help of Dirac delta function 2 . For example, if the cost of a Web service is $M$, then $f(q)=\delta(q-M)$. If the

$\overline{2} \delta(x)$ is the Dirac delta function. $\delta(x)=+\infty$ when $x=0$ and $\delta(x)=0$ when $x \neq 0$. 
cost of a Web service is $N_{1}$ with a probability of $p_{1}$ and $N_{2}$ with a probability of $p_{2}\left(p_{1}+p_{2}=1\right)$, then the distribution of this Web service can be expressed as $f(q)=p_{1} \delta\left(q-N_{1}\right)+p_{2} \delta\left(q-N_{2}\right)$.

QoSSum. Computing the PDF of the QoSSum of two component QoS distributions is a problem of deducing the PDF of the sum of independent variables, which is the convolution of each of their density functions [10],

$$
f(q)=f_{1}(q) \circledast f_{2}(q)=\left(f_{1} * f_{2}\right)(q)=\int_{\eta=0}^{q} f_{1}(\eta) f_{2}(q-\eta) d \eta
$$

where $f(q)$ is the PDF of the QoS of a composition pattern, $f_{1}(q)$ and $f_{2}(q)$ are the PDFs of the component services.

Let us take a simple example of the execution time of a Sequential Pattern with two component services. The PDFs of the execution time of the two component services are $f_{1}(t)$ and $f_{2}(t)$ respectively. The probability for the execution time of the first service being $\tau(\tau \in(0, t))$ and the second service being $t-\tau(t \in$ $(0,+\infty))$ is $f_{1}(\tau) f_{2}(t-\tau)$. Therefore, the probability for the Sequential Pattern being finished at time $t$ is the integral of $f_{1}(\tau) f_{2}(t-\tau)$ over $(0, t)$ where $\tau$ is the variable, i.e. $f(t)=\int_{\tau=0}^{t} f_{1}(\tau) f_{2}(t-\tau) d \tau$. The result is the same as what we get from Formula (11).

QoSMin. The probability distribution of the QoSMin of $n$ component QoS distributions is the distribution of the minimum of $n$ independent variables which can be calculated as:

$$
F(q)=F_{1}(q) \otimes \ldots \otimes F_{i}(q) \otimes \ldots \otimes F_{n}(q)=1-\prod_{i=1}^{n}\left[1-F_{i}(q)\right]
$$

where $F(q)$ is the CDF of the QoS of a composition pattern; $n$ is the number of component services within this pattern; and $F_{i}(q)$ is the CDF of the QoS of component service $i$.

Then the PDF can be obtained by differentiating both sides of Equation (2) with respect to $q$ :

$$
f(q)=f_{1}(q) \otimes \ldots \otimes f_{i}(q) \otimes \ldots \otimes f_{n}(q)=\sum_{i=1}^{n} f_{i}(q) \prod_{j=1, \ldots, n \& j \neq i}\left[1-F_{j}(q)\right]
$$

where $f(q)$ is the PDF of a composition pattern; $n$ is the number of component services; $f_{i}(q)$ is the PDF of component service $i$; and $F_{j}(q)$ is the CDF of component service $j$.

Let us take a QoS metric, response time as an example. Assume that $X$ and $Y$ are two Web services in a Parallel Pattern with single merge. The probabilities for them to be finished within time $t$ are $F_{X}(t)$ and $F_{Y}(t)$ respectively. The probability for neither of them being able to finish within time $t$ is $\left(1-F_{X}(t)\right)(1-$ $F_{Y}(t)$ ), therefore, the probability for either of them being able to finish within time $t$ is $1-\left(1-F_{X}(t)\right)\left(1-F_{Y}(t)\right)$. The fact that at least one of the Web services 
can be finished within $t$ means that $t$ is the shorter execution time of the two Web services.

QoSMax. The distribution of the QoSMax of $n$ component QoS distributions is the distribution of the maximum of $n$ independent variables which can be calculated as:

$$
F(q)=F_{1}(q) \otimes \ldots \otimes F_{i}(q) \oslash \ldots \otimes F_{n}(q)=\prod_{i=1}^{n} F_{i}(q)
$$

where $F(q)$ is the CDF of the QoS of a composition pattern; $n$ is the number of component services within this pattern; and $F_{i}(q)$ is the CDF of the QoS of component service $i$.

The PDF can be obtained by differentiating both sides of Equation (4) with respect to $q$ :

$$
f_{(q)}=f_{1}(q) \otimes \ldots \otimes f_{i}(q) \otimes \ldots \otimes f_{n}(q)=\sum_{i=1}^{n} f_{i}(q) \prod_{j=1, \ldots, n \& j \neq i} F_{j}(q)
$$

where $f(q)$ is the PDF of a composition pattern; $n$ is the number of component services within this pattern; $f_{i}(q)$ is the PDF of component service $i$; and $F_{j}(q)$ is the CDF of component service $j$.

Let us take execution time as an example. Assume that $X$ and $Y$ are two concurrently running Web services in a Parallel Pattern with synchronized merge. The probability for $X$ and $Y$ to be finished within time $t$ is $F_{X}(t)$ and $F_{Y}(t)$ respectively. Therefore, the probability for both of them to be finished within time $t$ is $F_{X}(t) F_{Y}(t)$. The fact that both Web services can be finished within $t$ means that $t$ is the longer execution time of the two Web services.

QoSWeightedSum. The QoS distribution for the QoSWeightedSum of component QoS distributions can be calculated as

$$
f(q)=f_{1}(q) \odot \ldots \odot f_{i}(q) \odot \ldots \odot f_{n}(q)=\sum_{i=1}^{n} p_{i} f_{i}(q)
$$

where $f(q)$ is the PDF of a composition pattern; $n$ is the number of component services within this pattern; $f_{i}(q)$ is the PDF of component service $i$; and $p_{i}$ is the execution probability for component service $i$.

Here we can take execution time as an example. Assume $X$ and $Y$ are two Web services within a Conditional Pattern with the execution probabilities being $p_{1}$ and $p_{2}$ respectively. The probabilities for $X$ and $Y$ to be finished at time $t$ are $f_{X}(t)$ and $f_{Y}(t)$ respectively. Therefore, the probability for the path of $X$ to be finished at time $t$ is $p_{1} f_{X}(t)$ and for the path of $Y$ to be finished at time $t$ is $p_{2} f_{Y}(t)$. Therefore, the probability for the Conditional Pattern to be finished at time $t$ is $f(t)=p_{1} f_{X}(t)+p_{2} f_{Y}(t)$.

QoS Probability Distribution Calculation for Composition Patterns. So far, we have discussed the operations and formulae involved in computing 
composite QoS distributions. Next, we will explain how component QoS distributions are aggregated for different composition patterns. Here, QoS metrics cost and time (execution time or response time) will be discussed as examples.

For a composition pattern with two component services, assume the probability distribution of the composite $\mathrm{QoS}$ is $c(q)$ for cost, $t(q)$ for time, and the probability distributions of component QoS are $c_{1}(q)$ and $c_{2}(q)$ for $\operatorname{cost}, t_{1}(q)$ and $t_{2}(q)$ for time. According to Table 2 , there are:

- in a Sequential Pattern: $c(q)=c_{1}(q) \circledast c_{2}(q) ; t(q)=t_{1}(q) \circledast t_{2}(q)$.

- in a Parallel Pattern with synchronized merge: $c(q)=c_{1}(q) \circledast c_{2}(q) ; t(q)=t_{1}(q) \otimes t_{2}(q)$.

- in a Parallel Pattern with single merge: $c(q)=c_{1}(q) \circledast c_{2}(q) ; t(q)=t_{1}(q) \otimes t_{2}(q)$.

- in a Conditional Pattern: $c(q)=c_{1}(q) \odot c_{2}(q) ; t(q)=t_{1}(q) \odot t_{2}(q)$.

- in a Loop Pattern:

The QoS computation for Loop Patterns is more complicated than other patterns. Next, we will discuss it in detail.

In [17, we have given detailed discussion on the structure analysis method to compute the QoS for an arbitrary Loop Pattern whose component QoS are fixed constant values. To sum up the method in [17], statistically, a Loop Pattern can be seen as a Conditional Pattern with a Sequential Pattern in each path. With the formula for calculating the execution probability of each path of the Conditional Pattern given in [17] (see Formula 7) and the formulae of computing the QoS of a Sequential Pattern and Conditional Pattern given in this paper, the distribution of the QoS of a Loop Pattern can be computed.

$$
p_{\text {path }_{l i}}=\left(\prod_{k=1}^{n} p_{k}\right)^{l}\left(\prod_{k=0}^{i-1} p_{k}\right)\left(1-p_{i}\right)
$$

where $p_{k}$ is the transition probability from vertex $v_{k}$ to $v_{k+1}$ and $p_{k}=1$ when $k=0, l$ is the number of times that the Loop is executed, $n$ is the number of vertices in the Loop, and $i$ is the index of the vertex where the Loop is jumped out of.

To compute the QoS distribution for a Loop Pattern, we can set a threshold value, $T H$, for $p_{\text {path }}$. When $p_{\text {path }}<T H$, the probability for the loop still being run is quite small. Therefore, the execution path with a probability smaller than $T H$ can be ignored. It means that $l=L$ times of looping is enough if $L$ satisfies $\left(\prod_{k=1}^{n} p_{k}\right)^{L}\left(\prod_{k=0}^{i-1} p_{k}\right)\left(1-p_{i}\right)<T H$.

The transition probability for each outgoing arc of a Loop Pattern (i.e. $p_{i j}$ in Figure $1(\mathrm{~d})$ where $i=1, \ldots, n$ and $j=1, \ldots, m_{i}$ ) has to be changed accordingly. Detailed formula on calculating the probabilities of the outgoing arcs can be found in our previous work [17. Then, the Loop Pattern can be replaced by one vertex. 


\section{Experiment}

In this section, experiments have been done to compare the performance of the proposed QoS calculation method (referred to as calculation method) with simulation method. In a simulation method, the execution of a composite service is simulated by exploring the Service Graph of the composite service. One single value for per QoS metric of the composite service is obtained for each run of a simulation by aggregating the QoS of each vertex that has been visited during the exploration of the Service Graph. After running the simulation for a number of times, a QoS sample (containing all the simulated QoS) for the composite service can be obtained. This QoS sample can be used to generate the QoS probability distribution for a composite service.

\subsection{Validation}

First, we shall test the accuracy of the calculation method and the simulation method mentioned earlier.

A composite service and its component services (see Figure 21) are deployed. Experiments have been done to monitor the QoS of the deployed composite service. The monitored QoS are referred to as experimental result. By comparing the composite QoS obtained by the simulation method and the calculation method (referred to as simulation result and calculation result respectively) with experimental result, the accuracy of the simulation method and the calculation method can be verified. We only consider the QoS metric execution time in the experiments.

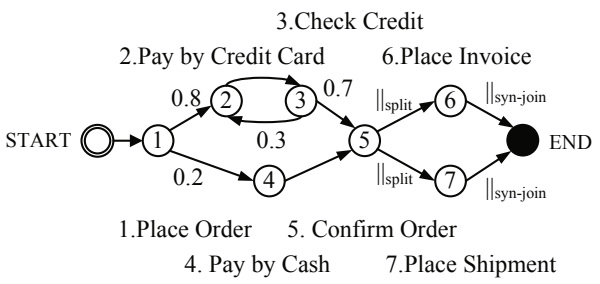

Fig. 2. An Example of A Service Graph

The seven component Web services in Figure 2 are developed and deployed on Apache Tomcat 5.5 server. Their execution time distributions follow the distributions in Figure 䢺. The BPEL process executing the composite service in Figure 2 is developed and deployed on an Active BPEL engine. The detailed information on service deployment is as follows:

(1) The simulation of the QoS probability distribution for a component Web service: An array containing 10000 values whose distribution conforms to the probability distribution of the Web service is generated and stored in a file. For each execution, the Web service will randomly read one value from the file and suspend for the indicated amount of time before it sends out a response.

\footnotetext{
3 These distributions are generated manually.
} 


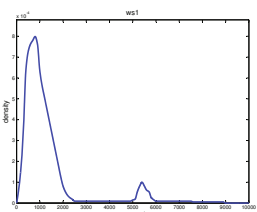

(a) WS1

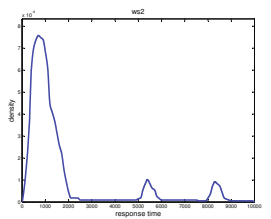

(b) WS2

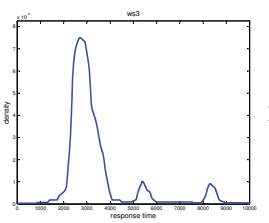

(c) WS3

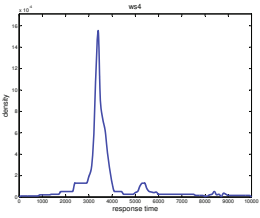

(d) WS4

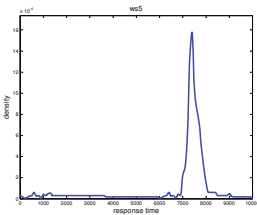

(e) WS5

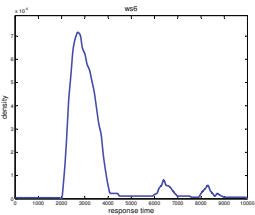

(f) WS6

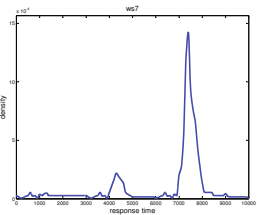

(g) WS7

Fig. 3. Probability Distributions of execution time

(2) The simulation of the transition probabilities within a composite service: A random number generator conforming to a uniform distribution is used. At component service 1 Place Order, a random number is generated and compared with 0.8. If it is smaller than 0.8, the output of service 1 is "Credit Card"; otherwise, the output is "Cash". At service 3 Check Credit, if the generated number is smaller than 0.7, the output is "Approved"; otherwise, it is "Disapproved".

(3) Experimental result: The developed composite service is invoked for 10, 000 times. For each invocation, an execution time is recorded. A histogram, shown in Figure 4, is generated based on the recorded data sample.

(4) Simulation result: Simulation result is in the form of a sample. To distinguish the simulation result from the histogram of experimental result, the simulated QoS are shown as dot-dashed curves in Figure 4(a), i.e. we plot the probability densities at different execution time in Figure 4(a) instead of histogram bars.

(5) Calculation result: The calculation result is shown as dashed curves in Figure 4(b) by plotting the probability densities at different execution time.

It can be seen from Figure 4 that both the simulation result and the calculation result fit the experimental result very well. The accuracy of both methods has been verified.

\subsection{Efficiency}

Next, the efficiency of using calculation method and simulation method will be compared.

We perform tests on Mac OS X 10.6.6 with $1.86 \mathrm{GHz}$ Intel Core 2 Duo processor and 2 GB memory. Both the proposed QoS calculation and simulation methods are implemented using $\mathrm{C} / \mathrm{C}++$ language. We test the time spent on QoS estimation by calculation and simulation methods for Sequential Patterns, Parallel Patterns, Conditional Patterns, and Loop Patterns respectively. The results are plotted by Matlab and shown in Figures 5, 6, 7, and 8 respectively. The 


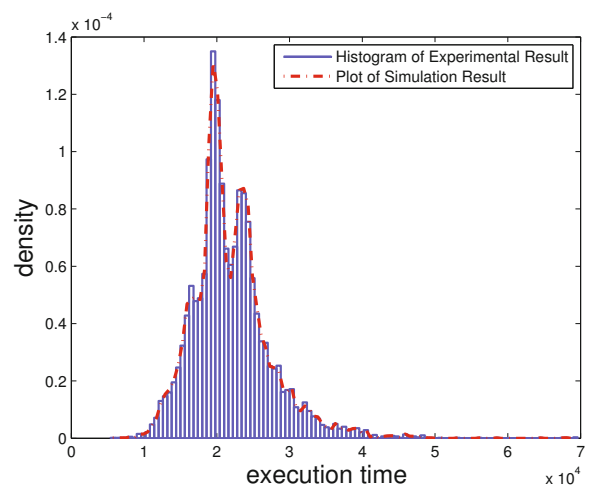

(a) Experimental and Simulation Results

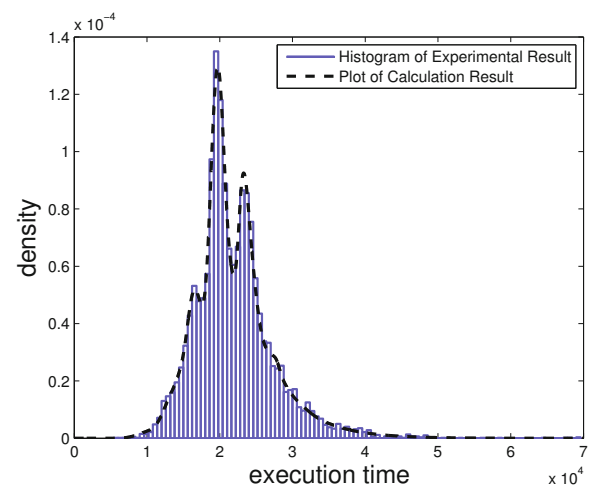

(b) Experimental and Calculation Results

Fig. 4. Results of Validation

$\mathrm{x}$-axis represents the number of component services in a composite service and the $\mathrm{y}$-axis represents the time (in $\mu \mathrm{s}$ ) spent on estimating the QoS distribution for a composite service. As the time spent on calculation method is significantly shorter than simulation method, we present the computation time of different methods in logarithmic scale. In each of Figures 5, 6, 7, and 8, there are four dashed lines and two solid lines. The four dashed lines represent the time spent on simulation method when the simulation is run for 5000, 10000, 15000, and 20000 times respectively. The two solid lines represent the time spent on calculation method when the probability distribution of each component QoS has 512 and 1024 bins respectively. One thing is to be noted: the time spent by simulation method changes irregularly for any Loop Patterns. This is because in the experiment, the transition probabilities in the Loop Pattern, the number of component services that can jump out of the Loop Pattern, the component services that jump out of the Loop Pattern, and the jumping out probabilities all change randomly when the number of component services changes.

Based on the performance comparison between calculation and simulation methods, it can be seen that the proposed QoS calculation method is far more

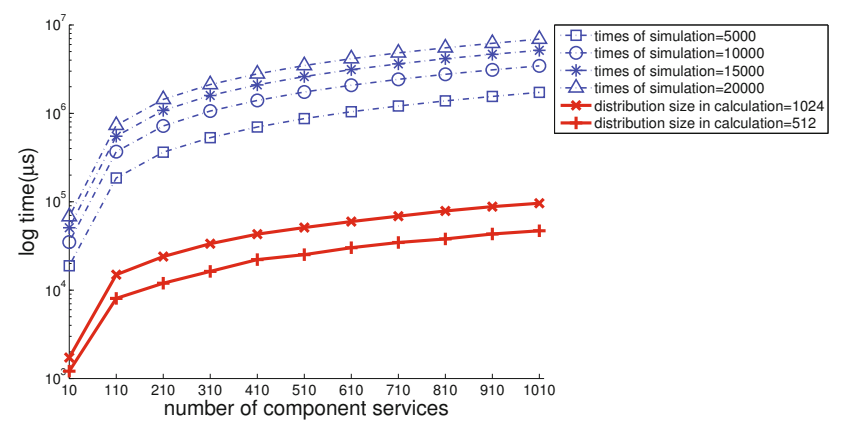

Fig. 5. Performance Comparison - Sequential Pattern 


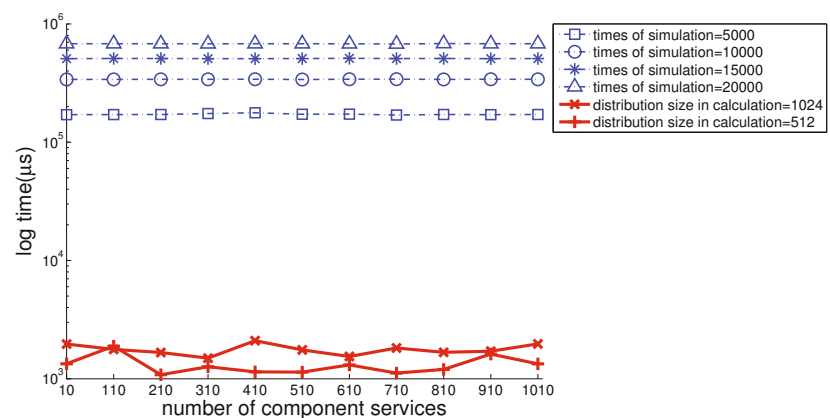

Fig. 6. Performance Comparison - Parallel Pattern

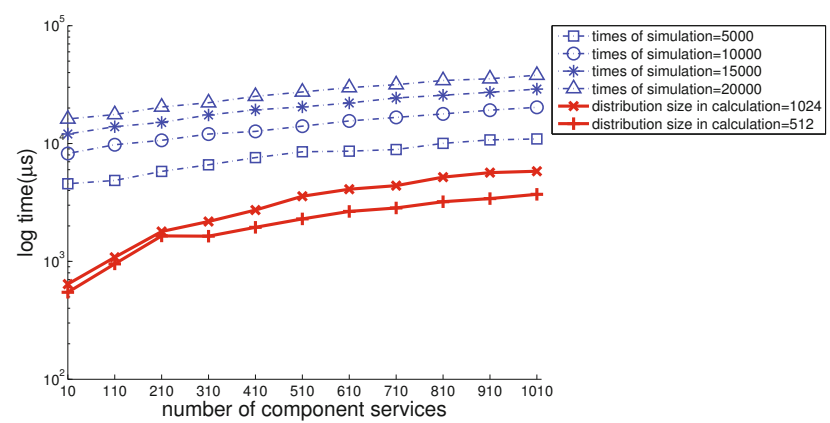

Fig. 7. Performance Comparison - Conditional Pattern

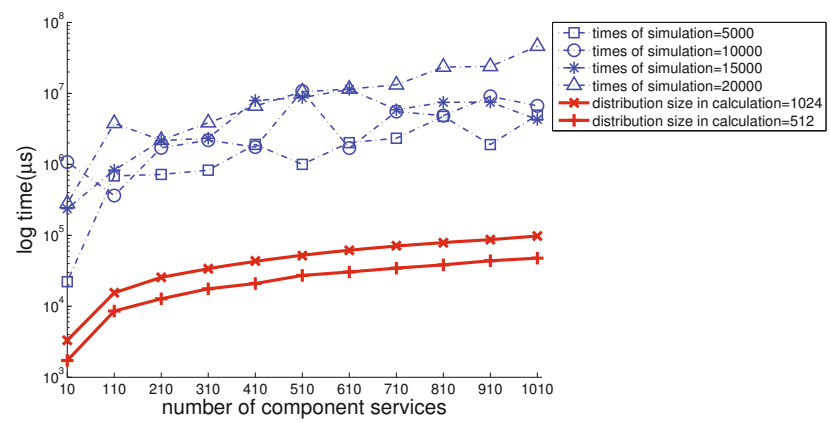

Fig. 8. Performance Comparison - Loop Pattern

efficient and outperforms simulation method in terms of computing QoS for all the basic composition patterns.

\section{Conclusion}

In this paper, we propose a systematic approach to calculate the QoS probability distribution for composite services. Experimental results show that the proposed QoS calculation method is far more efficient than existing method and can be used in real-time scenarios. 
The proposed method is based on the assumption that the QoS of component services are independent of each other, which is not always the case in reality. Research will be done to relax this assumption to make the proposed QoS aggregation method more robust to fit into any environment.

\section{References}

1. Artaiam, N., Senivongse, T.: Enhancing service-side qos monitoring for web services. In: ACIS, pp. 765-770 (2008)

2. Benatallah, B., Sheng, Q.Z., Ngu, A.H.H., Dumas, M.: Declarative composition and peer-to-peer provisioning of dynamic web services. In: ICDE, pp. 297-308 (2002)

3. Cardoso, J., Miller, J., Sheth, A., Arnold, J.: Quality of service for workflows and web service processes. Journal of Web Semantics 1, 281-308 (2004)

4. Casati, F., Ilnicki, S., Jin, L., Krishnamoorthy, V., Shan, M.-C.: Adaptive and Dynamic Service Composition in eFLOW. In: Wangler, B., Bergman, L.D. (eds.) CAiSE 2000. LNCS, vol. 1789, pp. 13-31. Springer, Heidelberg (2000)

5. Comuzzi, M., Pernici, B.: A framework for qos-based web service contracting. TWEB 3(3), 10:1-10:52 (2009)

6. Hwang, S.Y., Wang, H., Tang, J., Srivastava, J.: A probabilistic approach to modeling and estimating the qos of web-services-based workflows. Inf. Sci. 177(23), 5484-5503 (2007)

7. Jaeger, M., Rojec-Goldmann, G., Muhl, G.: Qos aggregation for web service composition using workflow patterns. In: EDOC, pp. 149-159 (2004)

8. Klein, A., Ishikawa, F., Honiden, S.: Efficient QoS-Aware Service Composition with a Probabilistic Service Selection Policy. In: Maglio, P.P., Weske, M., Yang, J., Fantinato, M. (eds.) ICSOC 2010. LNCS, vol. 6470, pp. 182-196. Springer, Heidelberg (2010)

9. Mani, A., Nagarajan, A.: Understanding Quality of Service for Web Services. IBM Software labs, India

10. Papoulis, A.: Probability, random variables, and stochastic processes. McGraw-Hill, New York (1965)

11. Rosario, S., Benveniste, A., Haar, S., Jard, C.: Probabilistic qos and soft contracts for transaction-based web services orchestrations. IEEE Transactions on Services Computing 1(4), 187-200 (2008)

12. Rosenberg, F., Platzer, C., Dustdar, S.: Bootstrapping performance and dependability attributes of web services. In: ICWS, pp. 205-212 (2006)

13. Yang, J., Papazoglou, M.P.: Service components for managing the life-cycle of service compositions. Inf. Syst. 29(2), 97-125 (2004)

14. Zeng, L., Benatallah, B., Ngu, A., Dumas, M., Kalagnanam, J., Chang, H.: Qosaware middleware for web services composition. IEEE Transactions on Software Engineering 30(5), 311-327 (2004)

15. Zheng, H., Yang, J., Zhao, W.: Qos analysis and service selection for composite services. In: SCC, pp. 122-129 (2010)

16. Zheng, H., Yang, J., Zhao, W.: Qos probability distribution estimation for web services and service compositions. In: SOCA, pp. 1-8 (2010)

17. Zheng, H., Zhao, W., Yang, J., Bouguettaya, A.: Qos analysis for web service composition. In: SCC, pp. 235-242 (2009)

18. Zheng, Z., Zhang, Y., Lyu, M.R.: Distributed qos evaluation for real-world web services. In: ICWS, pp. 83-90 (2010) 